\title{
Carcinoma mucinoso invasor da mama e seus diagnósticos diferenciais em biópsia por agulha grossa: revisão da literatura
}

\author{
Mucinous invasive carcinoma of the breast and its differential diagnosis by core biopsy: review of the literature
}

Juliana Silva Zanetti'; Alfredo Ribeiro-Silva²

abstract

The needle core biopsy is a technique applied to remove small cylinders of breast tissue. The development of accurate radiological techniques for location of breast lesions has spread the use of core biopsy as the first histological approach to non-palpable lesions. The differential diagnosis of mucinous carcinoma and benign mucinous lesions by core biopsy may be challenging, mainly when the lesion shows mucin extravasation. The accuracy of diagnosis in these cases is extremely important to determine the type of procedure to be performed, as well as the treatment choice. This study shows a review and an update of the literature as to invasive mucinous carcinoma of the breast and its differential diagnosis, with emphasis on the challenges of diagnosis by core biopsy. Among the differential diagnoses are fibrocystic changes with luminal mucin, mucinous papillary lesions, mucocele-like lesions that range from benign to those associated with atypical ductal hyperplasia and ductal carcinoma in situ. Mucinous changes may also be found in a variety of lesions such as fibroadenoma, phyllodes tumor, pleomorphic adenoma and nodular mucinosis. In conclusion, core biopsy is a reliable technique for the diagnosis of mucinous carcinoma of the breast and its differential diagnosis, however, in doubtful cases or when the sample is scarce, it is advisable to perform an excisional biopsy to clarify the diagnosis.

\footnotetext{
\begin{tabular}{|c|c|}
\hline $\mathbf{u}$ & Imo \\
\hline $\begin{array}{l}\text { Mama } \\
\text { Biópsia por agulha grossa } \\
\text { Carcinoma mucinoso } \\
\text { Lesões mucinosas } \\
\text { Diagnóstico diferencial }\end{array}$ & $\begin{array}{l}\text { A biópsia por agulha grossa (BAG), ou core biopsy, é uma técnica utilizada para retirar pequenos cilindros } \\
\text { de tecido mamário. Além de lesões palpáveis, o desenvolvimento de técnicas radiológicas acuradas de } \\
\text { localização de lesões mamárias difundiu o uso da BAG como primeira abordagem histológica de lesões } \\
\text { não palpáveis. O diagnóstico diferencial do carcinoma mucinoso com lesões mucinosas benignas por } \\
\text { BAG pode ser desafiador, principalmente se a lesão apresentar extravasamento de mucina. A acurácia } \\
\text { do diagnóstico nesses casos é de extrema relevância para determinar o tipo de procedimento a ser } \\
\text { realizado e o tratamento a ser seguido. Este estudo traz revisão e atualização da literatura sobre } \\
\text { carcinoma mucinoso invasor da mama e seus diagnósticos diferenciais, com ênfase nos desafios para } \\
\text { diagnóstico por intermédio da BAG. Entre os diagnósticos diferenciais estão alterações fibrocísticas } \\
\text { com mucina luminal, lesões mucinosas papilares e mucocele-símile (que variam desde as benignas até } \\
\text { aquelas associadas a hiperplasia ductal atípica e carcinoma ductal in situ). Alterações mucinosas também } \\
\text { podem ser encontradas em uma variedade de lesões, como fibroadenoma e tumor phyllodes, adenoma } \\
\text { pleomórfico e mucinose nodular. Conclui-se que a BAG é uma técnica confiável para diagnóstico de } \\
\text { carcinoma mucinoso da mama e seus diagnósticos diferenciais, porém, em casos de dúvida ou de } \\
\text { escassez de material é prudente realizar biópsia excisional para melhor esclarecimento do diaqnóstico }\end{array}$ \\
\hline
\end{tabular}

unitermos

Carcinoma mucinoso

Lesões mucinosas

Diagnóstico diferencial
}

key words

Breast

Core biopsy

Mucinous carcinoma

Mucinous lesions

Differential diagnosis 


\section{Introdução}

O diagnóstico de lesões mucinosas da mama é controverso, principalmente quando há extravasamento de mucina. A acurácia do diagnóstico nesses tipos de lesão é de extrema relevância por determinar o tipo de procedimento a ser realizado e o tratamento a ser seguido. Atualmente, a biópsia por agulha grossa (BAG) é uma técnica muito utilizada devido a precisão e confiabilidade. O presente estudo traz revisão e atualização da literatura sobre carcinoma mucinoso da mama e seus diagnósticos diferenciais, com ênfase nos desafios para diagnóstico por intermédio da BAG.

\section{Lesões mucinosas mamárias}

Mucinas são carboidratos complexos secretados principalmente por células epiteliais especializadas ${ }^{(20)}$ e do tecido conjuntivo. São os principais constituintes do muco normal(25). É conhecida por desempenhar papel protetor dos tecidos compostos por células epiteliais. Além disso, acredita-se que está envolvida em diversos processos, como diferenciação epitelial, modulação da adesão celular e sinalização celular ${ }^{(22)}$. A primeira mucina clonada foi MUC-1, a partir de tecido neoplásico da mama ${ }^{(19,25)}$ e células tumorais pancreáticas ${ }^{(25)}$. Posteriormente foi identificada MUC-2, originalmente clonada a partir de células epiteliais intestinais ${ }^{(25)}$. MUC-2 e MUC-5 possuem propriedades físicoquímicas que favorecem a formação do aspecto coloide no carcinoma mucinoso ${ }^{(19,20)}$.

As lesões mucinosas são descritas pela presença de mucina, e essas lesões podem estar relacionadas com carcinoma ductal in situ micropapilar. Nem todas as lesões mucocelesímile são acompanhadas por processos neoplásicos ${ }^{(22)}$. A patogênese dessas lesões permanece desconhecida, mas acredita-se que pode ser decorrente de secreção excessiva de mucina ou obstrução ductal ${ }^{(14)}$.

Lesões de mama que apresentam mucina incluem alterações fibrocísticas com mucina luminal, lesões mucinosas papilares, carcinoma mucinoso e lesões mucocele-símile, que variam desde as benignas até aquelas associadas a hiperplasia ductal atípica e carcinoma ductal in situ. Alterações mucinosas também podem ser encontradas em uma variedade de lesões, como fibroadenoma e tumor phyllodes, adenoma pleomórfico e mucinose nodular ${ }^{(22)}$. Na Tabela são apresentados alguns exemplos de lesões mamárias contendo mucinas extracelular, intracelular e estromal. Em vista da relação com hiperplasia ductal atípica ou carcinoma ductal in situ, é recomendada cuidadosa análise das lesões mucocele-símile da mama(27).

Tumores mucocele-símile foram descritos pela primeira vez por Rosen, em 1986, como lesões benignas caracterizadas por múltiplos cistos rotos com conteúdo mucinoso extravasado no estroma adjacente ${ }^{(6,14,22)}$. Já os da mama são neoplasias raras e, embora de início tenham sido considerados exclusivamente benignos, hoje sabe-se que podem evoluir para carcinoma mucinoso. Esses tumores estão sendo cada vez mais relatados na literatura, estando relacionados com hiperplasia ductal e carcinoma. Além disso, eles têm sido considerados espectro de lesões patológicas, que incluem tumores benignos, hiperplasia ductal atípica, carcinoma ductal in situ e mucinoso(6).

Entre as lesões da mama relacionadas com mucina estromal está o fibroadenoma com estroma mixoide. O estroma mucinoso pode ser encontrado também em tumores phyllodes, adenoma pleomórfico, carcinomas adenoide cístico e metaplásico. Mucinose nodular de mama é uma lesão extremamente rara, geralmente presente na região subareolar de mulheres jovens, e patologicamente caracterizada como massa mixoide ${ }^{(22)}$. O cistadenocarcinoma

Tabela Tipos de lesão mamária contendo mucinas extracelular, intracelular e estromal

\begin{tabular}{lll}
\hline Mucina extracelular & Mucina intracelular & Mucina estromal \\
Alteração fibrocística com mucina luminal & Metaplasia mucinosa & Fibroadenoma \\
$\begin{array}{l}\text { Lesões mucocele-símile benignas } \\
\text { Lesões mucocele-símile com hiperplasia ductal } \\
\text { atípica }\end{array}$ & Neoplasia lobular & Tumor phyllodes \\
$\begin{array}{l}\text { Lesões mucocele-símile com carcinoma ductal } \\
\text { in situ }\end{array}$ & Carcinoma ductal in situ & Adenoma pleomórfico \\
$\begin{array}{l}\text { Lesões mucocele-símile com carcinoma lobular invasivo } \\
\text { mucinoso invasivo }\end{array}$ & Carcinoma de células escamosas \\
& Carcinoma ductal invasivo & Carcinoma metaplásico produtor \\
& & de matriz \\
\hline
\end{tabular}


mucinoso é uma lesão de mama muito incomum, com poucos casos relatados na literatura, e que pode mimetizar lesões mucocele-símile ${ }^{(22)}$.

\section{Carcinoma mucinoso da mama}

Descrito pela primeira vez em 1826, foi considerado, na época, um achado patológico incomum ${ }^{(12)}$. A incidência desse tipo de carcinoma, também conhecido como coloide, mucoso, mucoide ou gelatinoso, corresponde a cerca de $1 \%$ a $2 \%$ de todos os carcinomas mamários $(7,15,18)$.

Caracterizado por abundante acúmulo de muco extracelular excretado pelas células tumorais ${ }^{(17)}$, o carcinoma mucinoso é encontrado geralmente em mulheres na pósmenopausa e é unilateral. Há apenas um relato na literatura que descreve um caso raro de carcinoma mucinoso mamário bilateral encontrado em mulher de 73 anos de idade(4).

No exame patológico, o carcinoma mucinoso é arredondado, bem circunscrito e com consistência gelatinosa ${ }^{(15)}$. Histologicamente é caracterizado por aglomerados de células arredondadas, por vezes isoladas, e cercadas por mucina extracelular (Figura) ${ }^{(28)}$. Ele é dividido em dois subtipos: puro e misto ${ }^{(12,17,18,28)}$. Para que um carcinoma mucinoso seja diagnosticado puro, é necessário que pelo menos $90 \%$ do tumor tenha componente mucinoso ${ }^{(9)}$. Caso tenha menos de $90 \%$, ele é considerado misto. Segundo a literatura, o carcinoma mucinoso puro raramente é espiculado e com microcalcificação, e, quando ocorre, é em menos de $5 \%$ dos $\operatorname{casos}^{(7)}$.

O carcinoma mucinoso invasivo tem poucas alterações genômicas comparado aos tipos histológicos mais comuns de câncer de mama. Até o momento não se encontrou correlação entre esse tipo de carcinoma e hereditariedade( ${ }^{(9)}$.

Com crescimento lento, carcinomas mucinosos puros são bem diferenciados e raramente se disseminam pelos linfonodos axilares (até 12\%). Já os mistos apresentam metástases linfonodais e taxa de mortalidade de cerca de $30 \%{ }^{(7)}$. Monzawa et al. pesquisaram a taxa de metástase linfonodal axilar para ambos os subtipos de carcinoma mucinoso: puro, aproximadamente 14\% a 15\%; misto, aproximadamente $46 \%{ }^{(17)}$. Vale destacar que nesses carcinomas existe uma associação entre tamanho do tumor e metástases para linfonodos axilares(2).

Com relação ao prognóstico, o do carcinoma puro é melhor que o do misto(18, 22). A maioria dos carcinomas mucinosos tem grau nuclear 1 e, nesses casos, a sobrevida de 10 anos é superior a $80 \%$; já para grau 2, é de $60 \%$ a

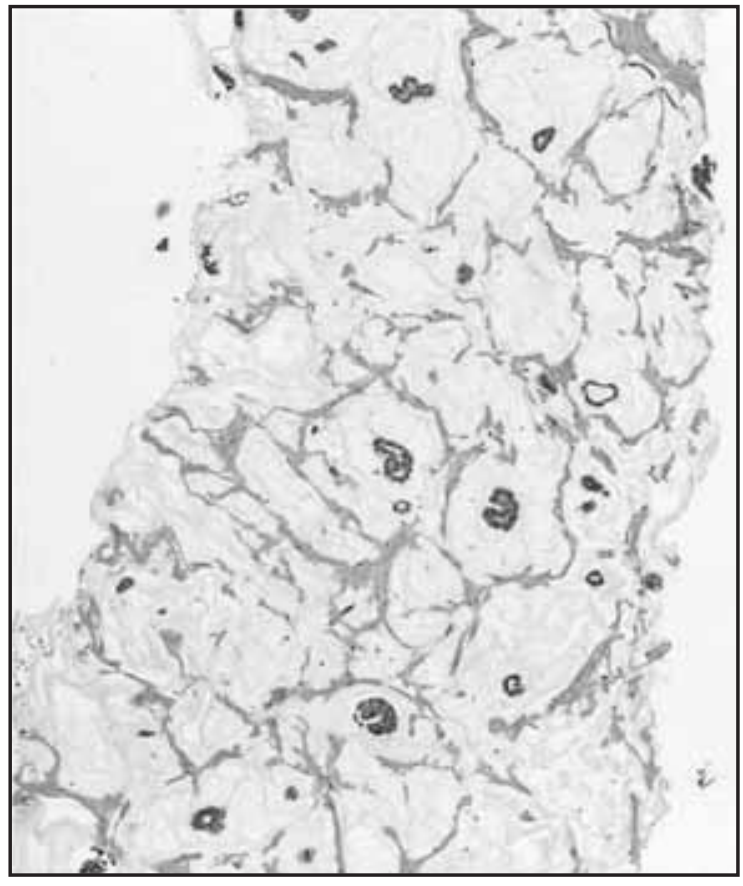

Figura A - Exemplo de carcinoma mucinoso invasor da mama diagnosticado em $B A G$. Observe o abundante muco extracelular permeando pequenos agrupamentos de células neoplásicas (HE, 100x)

BAG: biópsia por agulha grossa; HE: hematoxilina e eosina.

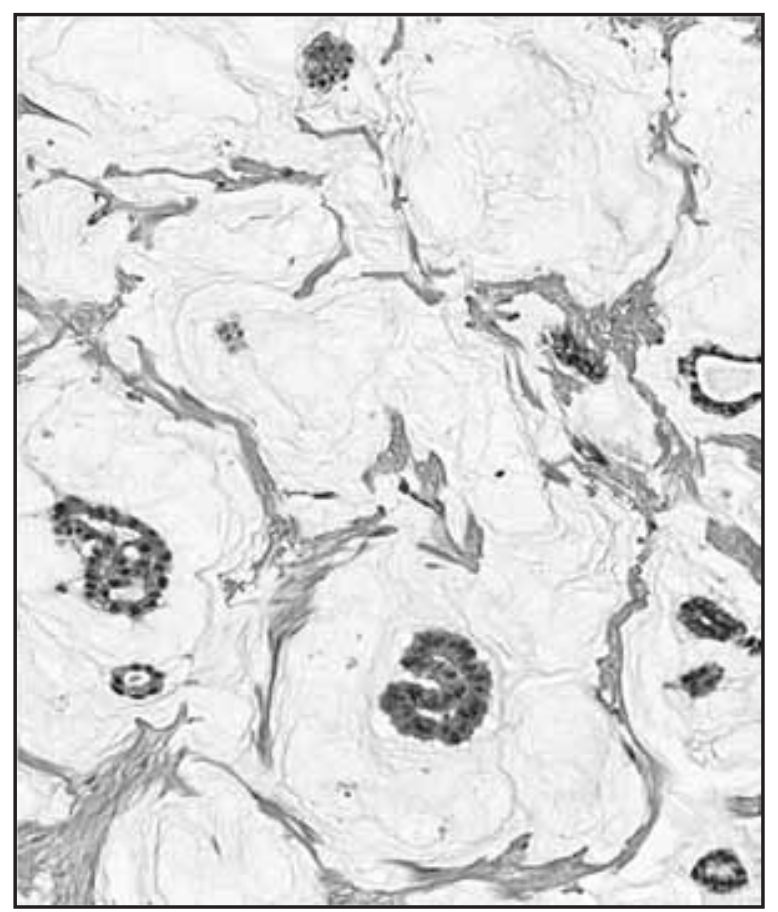

Figura B - Maior detalhe (400x) mostrando células neoplásicas, pequenas e uniformes, exibindo mínimo pleomorfismo nuclear

$80 \%{ }^{(22)}$. Cerca de $7 \%$ dos carcinomas de mama que ocorrem em mulheres acima de 75 anos de idade são mucinosos puros; em mulheres mais jovens ( 35 anos de idade) esse tipo de carcinoma corresponde somente a $1 \%{ }^{(7)}$. Yamaguchi et al. relatam um caso bastante incomum de uma mulher 
com 30 anos de idade na fase pré-menopausa que recebeu o diagnóstico de carcinoma mucinoso de mama de grau nuclear $2^{(26)}$.

Entre a família de genes MUC, o carcinoma mucinoso expressa predominantemente MUC-2 e MUC-6. MUC-2 é uma glicoproteína responsável pelo acúmulo de mucina extracelular que circunda as células epiteliais malignas e que atua como barreira à disseminação dessas células ${ }^{(22)}$. Essa é uma das teorias que tentam explicar porque carcinomas mucinosos estão associados a melhor prognóstico.

Esses carcinomas podem apresentar diferenciação neuroendócrina, a qual pode ser avaliada pelo método imunohistoquímico com os marcadores enolase, sinaptofisina e cromogranina ${ }^{(23)}$. Tse et al. classificaram os carcinomas mucinosos em três tipos, conforme grau de diferenciação: A (sem diferenciação), B (com diferenciação) e AB (forma intermediária entre $A$ e B). Embora o significado da diferenciação neuroendócrina não esteja bem estabelecido, existem evidências de que carcinomas mucinosos com essa diferenciação apresentam melhor prognóstico.

\section{Biópsia por agulha grossa}

Atualmente, com as campanhas de rastreamento do câncer de mama e o diagnóstico feito cada vez mais precocemente, tornou-se fundamental a identificação de lesões mamárias de dimensões muito reduzidas e com alta precisão quanto à sua localização. $\mathrm{O}$ desenvolvimento de técnicas altamente acuradas de localização de lesões mamárias por métodos radiológicos, estereotáxicos, ultrassonográficos, entre outros, tornou possível a utilização da punção aspirativa com agulha fina e da BAG para diagnóstico dessas lesões.

A primeira técnica utilizada foi a biópsia aspirativa por agulha fina, mas esse procedimento muitas vezes proporciona quantidade insuficiente de material para diagnóstico, gerando inúmeros casos de resultados falso-negativos, além de dificuldade na classificação das lesões ${ }^{(1)}$. Assim, na década de 1980, foi introduzida a técnica da biópsia por agulha grossa (core biopsy) juntamente com técnicas por controle de imagem. Isso permitiu maior acurácia no diagnóstico de lesões mamárias difíceis de serem identificadas e palpadas.

A BAG é utilizada para retirar pequenos cilindros de material de áreas contendo tecidos anormais. Em geral esse procedimento é realizado sob anestesia local e dentro do próprio consultório médico. Normalmente o médico utiliza guias para direcionar a agulha no interior da mama, enquanto ele vai palpando a mama à procura de nodulações e áreas diferenciadas. De início a BAG era realizada com agulhas de calibres 18 e 16, desenvolvidas para biópsia de próstata, mas as amostras eram inadequadas. Por isso desenvolveu-se a agulha de calibre 14, especialmente para a mama ${ }^{(11)}$.

Hoje a BAG é considerada uma técnica minimamente invasiva e altamente difundida e tem sido utilizada em larga escala por permitir a obtenção de melhor resultado quando comparado à biópsia de agulha fina ${ }^{(16)}$. Para a paciente, a BAG é um procedimento muito simples que permite identificação e esclarecimento da natureza do nódulo mamário, além de não deixar cicatriz nem alterar a estética da mama. No procedimento realizado com BAG obtêm-se fragmentos que podem representar amostra significativa da lesão mamária(1). Se o diagnóstico por agulha grossa for de benignidade, a cirurgia pode ser evitada, visto que a BAC apresenta diagnóstico confiável(1, 16).

A utilização da BAG permite a retirada de fragmentos de várias áreas anormais da mama e tem grande precisão no diagnóstico ( $97 \%$ a $100 \%$ de acurácia). Porém, se o nódulo for muito pequeno, essa técnica pode não ser eficaz, uma vez que irá fornecer escasso material para exame. Além de lesões pequenas, essa técnica é contraindicada a pacientes com próteses mamárias ou em casos de coagulopatias ${ }^{(16)}$. Dependendo do tamanho ou da localização inicial do nódulo mamário, a BAG pode ser feita de diversas maneiras. A freehand needle biopsy pode ser realizada quando há nódulo ou massa facilmente palpável, mas se for muito pequeno para ser sentido ou muito profundo, três outros métodos podem ser utilizados: BAG guiada por ultrassom, BAG estereotáxica e biópsia assistida a vácuo (ou mamotomia).

Em algumas instituições, a BAG é considerada tão confiável quanto à biópsia cirúrgica para confirmar se o diagnóstico de um nódulo é ou não canceroso. Quando comparada à biópsia de agulha fina, a BAG pode distinguir tumores benignos de malignos e formas in situ ou invasivas, além de permitir avaliação da histologia, dos fatores prognósticos e preditivos no câncer de mama, proporcionando assim melhor escolha do tratamento a ser desenvolvido(8).

Uma possível complicação da BAG é a implantação de células cancerosas no trajeto da agulha. Esse tipo de ocorrência é dado de acordo com o calibre da agulha utilizada, além do tipo histológico do tumor, chamando a atenção para alto risco entre carcinomas mucinosos e tumor phyllodes ${ }^{(10)}$.

Melo et al. mostraram que a BAG é uma intervenção muito útil para o diagnóstico de lesões mamárias suspeitas de malignidade. Nesse estudo, entretanto, os autores con- 
cluem que, em alguns casos, é necessária a realização da biópsia cirúrgica após a BAG. Entre esses casos destacam-se algumas lesões benignas, como lesão esclerosante radial (que pode estar associada a carcinoma lobular ou tubular), hiperplasia ductal atípica e casos em que é difícil diferenciar carcinoma ductal in situ do invasivo( ${ }^{(16)}$. Em caso de dúvidas quanto à natureza da amostra, deve-se realizar uma biópsia cirúrgica para confirmar o diagnóstico. El-Sayed et al. relataram que a excisão cirúrgica de lesões benignas muitas vezes é inevitável após a BAG, devido a escassez de tecido disponível para diagnóstico ${ }^{(8)}$.

\section{Lesões mucinosas mamárias e BAG}

Relatos de lesões mucinosas de mama diagnosticadas por BAG são incomuns na literatura. Esse diagnóstico pode ser desafiador principalmente se a lesão apresentar extravasamento de mucina. Segundo Wang et al. é necessário realizar exame cuidadoso de lesões mucocele-símile de mama por causa de sua relação com hiperplasia ductal atípica ou carcinoma ${ }^{(27)}$. Muitas lesões mucinosas da mama acabam sendo diagnosticadas erroneamente como carcinoma mucinoso. Dessas lesões, destacam-se alterações fibrocísticas com extravasamento de mucina e lesões mucocele-símile, que podem estar relacionadas com atipia epitelial, hiperplasia ductal atípica ou mesmo carcinoma in situ. As lesões mucocele-símile surgem quando ocorre ruptura de cistos formados por células epiteliais produtoras de muco, o qual pode dissecar o estroma, simulando malignidade. Nessas lesões existe resposta inflamatória mínima, e a mucina tende a se calcificar, formando massas lobuladas em exames de mamografia ou mesmo na palpação clínica.

Na lesão mucocele-símile sem atipias ("benigna"), observam-se múltiplos cistos repletos de muco, revestidos por células uniformes cuboidais ou colunares, e pode haver extravasamento desse muco para o estroma adjacente. Já a lesão mucocele-símile relacionada com hiperplasia ductal atípica exibe um cisto repleto de muco, revestido por uma população monótona de células epiteliais pequenas, redondas e com núcleo denso. Essa área de células monótonas não pode exceder $3 \mathrm{~mm}$, pois, se assim o for, o diagnóstico passa a ser de lesão mucocele-símile associada a carcinoma ductal in situ. Em ambos os casos não pode haver células epiteliais isoladas dispersas no muco, pois essa é uma característica dos carcinomas mucinosos invasores (Figura).

Wang et al. relataram que o diagnóstico dado pela BAG se manteve após a biópsia excisional em 91\% dos casos. Nesse estudo, dois casos diagnosticados como lesão mucocele-símile sem atipias mostraram, após a biópsia excisional, apenas alterações fibrocísticas sem resíduos de lesão mucocele-símile. Para carcinomas mucinosos, todos os casos diagnosticados pela BAG se mantiveram após a biópsia excisional. Os autores também afirmaram que, em raríssimos casos, a BAG pôde detectar apenas mucina sem atipia epitelial em pacientes com carcinoma mucinoso, mas que na prática isso é um evento de ocorrência rara ${ }^{(27)}$. Em 2008, Cheung et al. também realizaram trabalho comparativo entre os resultados da BAG e os da biópsia cirúrgica e observaram que a concordância entre eles é próxima a $100 \%{ }^{(5)}$. Verkooijen et al. realizaram estudo sobre a acurácia do diagnóstico da BAG estereotáxica em 871 lesões não palpáveis de mama. Em 13 casos o patologista não pôde realizar o diagnóstico histológico da lesão pela escassez de material fornecido pela BAG. Nesse estudo, o valor preditivo para o diagnóstico de lesões benignas foi de $96 \%{ }^{(24)}$.

Outros autores, como Carder et al., defendem que seja feita a biópsia excisional da lesão em todos os casos de BAG em que for identificada mucina no estroma ou no interior de ductos e/ou cistos. Isso porque, segundo esses autores, há risco de malignidade, principalmente se houver atipias associadas ou se a lesão apresentar-se como massa no exame de imagem ${ }^{(3)}$.

Quando comparados os diagnósticos feitos pela punção aspirativa por agulha fina e os da BAG, nota-se que a BAG, por permitir a retirada de maior quantidade de material, proporciona melhor resultado no diagnóstico de certos tipos de lesões mamárias. Lam et al. compararam os diagnósticos feitos pela punção aspirativa por agulha fina com a posterior biópsia cirúrgica e concluíram que as taxas de falso-positivo e falso-negativo com a punção aspirativa é de 1,1\% e 6,2\%, respectivamente. Isso os levou a concluir que, com a punção aspirativa, o carcinoma mucinoso de mama pode, devido à baixa celularidade, ser confundido com lesões benignas. No mesmo trabalho, os autores descreveram ainda que a BAG, para diagnóstico de carcinoma mucinoso de mama, tem resultado muito mais confiável quando comparada com a punção aspirativa por agulha fina ${ }^{(13)}$.

Conclui-se, então, que a biópsia por agulha grossa é uma técnica confiável para diagnóstico de carcinoma mucinoso da mama e seus diagnósticos diferenciais, porém, em casos de dúvida ou de escassez de material, é prudente realizar biópsia excisional para melhor esclarecimento do diagnóstico. 


\section{Referências}

1. ABREU-E-LIMA, M. C. C. et al. Sensibilidade e especificidade da core biopsy estereotática no diagnóstico histopatológico das lesões mamárias impalpáveis. Rev Ass Med Brasil, v. 45, n. 4, p. 290-4, 1999.

2. BARKLEY, C. R. et al. Mucinous breast carcinoma: a large contemporary series. Am J Surg, v. 196, n. 4, p. 549-51, 2008.

3. CARDER, P. J.; MURPHY, C. E.; LISTON, J. C. Surgical excision is warranted following a core biopsy diagnosis of mucocele-like lesion of the breast. Histopathology, v. 45, n. 2, p. 148-54, 2004.

4. CHANG, Y. W.; KWON, K. H.; LEE, D. W. Synchronous bilateral mucinous carcinoma of the breast: case report. Clin Imaging, v. 33, n. 1, p. 62-6, 2008.

5. CHEUNG, K. L. et al. Pathological features of primary breast cancer in the elderly based on needle core biopsies. A large series from a single centre. Crit Rev Oncol Hematol, v. 67, n. 3 p. 263-7, 2008.

6. $\mathrm{CHO}, \mathrm{J}$. S. et al. A mucocele-like tumor of the breast associated with ductal carcinoma in situ and mucinous carcinoma: a case report. J Breast Cancer, v. 12, n. 2, p. 117-20, 2009.

7. DHILLON, R. et al. Screen-detected mucinous breast carcinoma: potential for delayed diagnosis. Clin Radiol, v. 61, n. 5, p. 423-30, 2006.

8. EL-SAYED, M. E. et al. Predictive value of needle core biopsy diagnoses of lesions of uncertain malignant potential (B3) in abnormalities detected by mammographic screening. Histopathology, v. 53, n. 6, p. 650-7, 2008.

9. GADRE, S. A. et al. Neovascularization in mucinous ductal carcinoma in situ suggests an alternative pathway for invasion. Histopathology, v. 53, n. 5, p. 545-53, 2008.

10. ISHIZUNA, K. et al. A case of mucinous carcinoma of the breast in which needle tract seeding was diagnosed by preoperative diagnostic imaging. Case report. Breast Cancer, 2009. No prelo.

11. JUNIOR, A. F. L. A core biopsy estereotática no diagnóstico das lesões mamárias impalpáveis altamente suspeitas de malignidade (categoria mamográfica BI-RADS ${ }^{\circledR}$ 5): um estudo de correlação radiologia/anatomia patológica. Dissertação. Pró-reitoria para assuntos de pesquisa e pós-graduação (PROPESQ), Centro de Ciências da Saúde, Universidade Federal de Pernambuco, Recife, 2007.

12. KOMENAKA, I. K. Pure mucinous carcinoma of the breast. Am J Surg, v. 187, n. 4, p. 528-32, 2004.

13. LAM, W. W. et al. Role of fine needle aspiration and tru cut biopsy in diagnosis of mucinous carcinoma of breast - from a radiologist's perspective. Clin Imaging, v. 30, n. 1, p. 6-10, 2006.

14. LeIBMAN, A. J.; StAEGER, C. N.; ChARnEY, D. A. Mucocele-like lesions of the breast: mammographic findings with pathologic correlation. AJR Am J Roentgenol, v. 186, n. 5, p. 1356-60, 2006.

15. LINDA, A. et al. Unusual malignant tumors of the breast: MRI features and pathologic correlation. Eur J Radiol, v. X, p. 1-7, 2009.
16. MELO, A. L. etal. Estudo prospectivo de 100 casos de core biópsia dirigida por ultra-som e revisão da literatura. Radiol Bras, v. 36, n. 6, p. 339-44, 2003.

17. MONZAWA, S. et al. Mucinous carcinoma of the breast: MRI features of pure and mixed forms with histopathologic correlation. AJR Am J Roentgenol, v. 192, n. 3, p. 125-31, 2009.

18. MORAND, C. et al. Pure mucinous carcinomas of the breast: prognostic study including DNA flow cytometry. Cytometry Part B. Clin Citom, v. 76, n. 1, p. 56-62, 2009.

19. O'CONNELL, J. T. et al. Altered mucin expression is a field change that accompanies mucinous (colloid) breast carcinoma histogenesis. Human Pathology, v. 29, n. 12, p. 1517-23, 1998.

20. RAKHA, E. A. et al. Expression of mucins (MUC1, MUC2, MUC3, MUC4, MUC5AC and MUC6) and their prognostic significance in human breast cancer. Mod Pathol, v. 18, n. 10, p. 1295-304, 2005.

21. RENSHAW, A. A. Can mucinous lesions of the breast be reliably diagnosed by core needle biopsy? Am J Clin Pathol, v. 118, n. 6, p. 82-4, 2002.

22. TAN, P. H.; TSE, G. M. K.; BAY, B. H. Mucinous breast lesions: diagnostic challenges. J Clin Pathol, v. 61, n. 1, p. 11-9, 2008.

23. TSE, G. M. K. et al. Neuroendocrine differentiation in pure type mammary mucinous carcinoma is associated with favorable histologic and immunohistochemical parameters. Modern Pathology, v. 17, n. 3, p. 568-72, 2004.

24. VERKOOJIEN, H. M. Diagnostic accuracy of stereotactic large core needle biopsy for non palpable breast disease results of a multicenter prospective study with 95\% surgical confirmation. Int J Cancer, v. 99, n. 6, p. 853-9, 2002.

25. VOYNOW, J. A.; GENDLER, S. J.; ROSE, M. C. Regulation of mucin genes in chronic inflammatory airway diseases. Am J Respir Cell Mol Biol, v. 34, n. 6, p. 661-5, 2006.

26. YAMAGUCHI, J. et al. A case of mucinous carcinoma of the breast that demonstrated a good pathological response to neoadjuvant chemotherapy despite a poor clinical response. Breast Cancer, v. 13, n. 1, p. 100-3, 2006.

27. WANG, J. et al. Can core biopsy reliably diagnose mucinous lesions of the breast? Am J Clin Pathol, v. 127, n. 1, p. 124-7, 2007.

28. WOODHAMS, R. et al. Diffusion-weighted imaging of mucinous carcinoma of the breast: evaluation of apparent diffusion coefficient and signal intensity in correlation with histologic findings. AJR Am J Roentgenol, v. 193, n. 1, p. 260-6, 2009.

\begin{tabular}{l|l} 
& Endereço para correspondência \\
\hline Alfredo Ribeiro-Silva \\
Universidade de São Paulo \\
Faculdade de Medicina de Ribeirão Preto \\
Laboratório de Patologia Ginecológica e Mamária \\
Departamento de Patologia \\
Av. Bandeirantes, 3.900 \\
Monte Alegre - Ribeirão Preto-SP \\
CEP: 14049-900 \\
e-mail: arsilva@fmrp.usp.br
\end{tabular}

